\title{
Steady State Solutions of the Satsuma-Mimura Diffusion Equation
}

\author{
L. v. WOLFERSDORF
}

Die stationären Lösungen der Diffusionsgleichung von Satsuma und Mimura, werden durch Zurückführung auf eine gewöhnliche komplexe .Differentialgleichung erster Ordnung-abgeleitet.

Установиншеся решения диффузионного уравнения Сатсума и Мимура выводятся с помодью редукции на обыкновениое комплексное дифференциальнос уравнсние первого порлдка.

The steady state solutions of the diffusion equation of Satsuma and Mimura are derived by means of reducing to an ordinary complex differential equation of first order.

Introduction. In their papers [3-5] J. Satsuma and M. Murura investigated à class of nonlinear nonlocal diffusion equations involving singular integral terms. They developed an exact linearization method for these equations and derived some interesting particular solutions in explicit form by this method. In the present paper we rederive the steady state solutions of Satsuma and Mimura in systematic way by reducing the steady equations to complex differential equations of first order. We find that apart from an obvious invariant shifting transformation the steady state solutions found by Satsuma and Mimura are the only ones with a prescribed asymptotic behaviour at infinity. Besides we obtain new periodic steady state solutions for some corresponding equations with variable diffusion coefficient.

1. Coth-type kernel. At first we deal with the equation

where

$$
d u_{x x}-(T u \cdot u)_{x}=0, \quad d>0,
$$

$$
(T u)(x)=\frac{1}{2 \delta} \int_{-\infty}^{\infty} u(\xi) \operatorname{coth} \frac{\pi}{2 \delta}(\xi-x) d \xi, \quad \delta>0 .
$$

We are looking for sufficiently smooth solutions $u=u(x)$ of (1) having vanishing limits $u( \pm \infty)=0$ and $u_{x}( \pm \infty)=0$ as $x \rightarrow \pm \infty$. Let $w(z)=u(x, y)+\mathrm{i} v(x, y)$, $z=x+\mathrm{i} y$, be a holomorphic function in the strip $I I:-\infty<x<\infty, 0<y<\delta$ with Hölder continuous boundary values on $y=0$ and $y=\delta$, where $u(x, 0)=u(x)$ and

$$
u(x, \delta)=0 ; \quad-\infty<x<\infty,
$$

and there exist the uniform (with respect to $y \in[0, \delta]$ ) limits $v( \pm \infty)$ satisfying $v(+\infty)=-v(-\infty)$ and $v_{x}( \pm \infty)$ satisfying $v_{x}(+\infty)=v_{x}(-\infty)$ as $x \rightarrow \pm \infty$. Then (cf. $[6: \S 3.16]$ )

$$
w(z)=\frac{1}{2 \delta \mathrm{i}} \int_{-\infty}^{\infty} u(\xi) \operatorname{coth} \frac{\pi}{2 \delta}(\xi-z) d \xi \quad \text { in } \Pi
$$


with the boundary values $v(x, 0)=-(T u)(x)$. Therefore integrating equation (i), we obtain the boundary relation $d u_{x}+v u=0$ on $y=0$ and due to (2) also on $y=\delta$, i.e. we have the boundary conditions $\operatorname{Re}\left[d w^{\prime}-(\mathrm{i} / 2) w^{2}\right]=0$ on $y=0, y=\delta$ for the function (3). Hence this function obeys the differential equation

$$
w^{\prime}-\frac{\mathrm{i}}{2 d} w^{2}=\mathrm{i} K_{0} \quad \text { in } \Pi
$$

with a real constant $\bar{K}_{0}\left(=v_{x}( \pm \infty)+(1 / 2 d) v^{2}( \pm \infty)\right)$. We have to determine holomorphic solutions $w$ of (4) in $\Pi$, (Hölder) continuous in $\bar{\Pi}$, with the supposed behaviour at infinity and satisfying additionally the boundary condition (2).

As can be easily seen for $K_{0}=0^{\prime}$, no non-trivial solutions to (4) of such kind exist. Therefore putting $K_{0}=-(1 / 2 d) K_{1}^{2}$ with $K_{1} \neq 0$ real or purely imaginary, we get the differential equation

$$
\frac{w^{\prime}}{w^{2}-K_{1}^{2}}=\frac{\mathrm{i}}{2 d}
$$

with the general solution

$$
w(z)=K_{1} \frac{1+C \mathrm{e}^{\left(K_{1} / d\right) 1 z}}{1-C \mathrm{e}^{\left(K_{2} / d\right) 1 z}}, \quad C \text { a complex number. }
$$

The supposed behaviour for $w$ at infinity requires $K_{1}$ purely imaginary, i.e. $K_{1}=\mathrm{i} d K$, $\mathcal{R}>\mathbf{0}$, and

$$
w(z)=\mathrm{i} d K \frac{1+\varrho \mathrm{e}^{l x-K z}}{1-\varrho \mathrm{e}^{1 \alpha-K z}},
$$

where we put $C=\varrho \mathrm{e}^{1 \dot{\alpha}}$ with $\varrho>0$ and $\alpha$ arbitrary real. From (5) there follows

and

$$
u(x, 0)=\frac{-2 d K \varrho \sin \alpha \mathrm{e}^{-K x}}{1+\varrho^{2} \mathrm{e}^{-2 K x}-2 \varrho \mathrm{e}^{-K x} \cos \alpha}
$$

$$
u(x, \delta)=\frac{-2 d K \varrho \sin (\alpha-K \delta) \mathrm{e}^{-K x}}{1+\varrho^{2} \mathrm{e}^{-2 K x}-2 \varrho \mathrm{e}^{-K x} \cos \alpha} .
$$

Therefore (2) implies that $\alpha=K \delta$ or $\alpha=K \delta+\pi$. Further it remains to secure the analyticity of $w$ in $\Pi$ and its continuity in $\bar{\Pi}$. This demands that $1-\varrho e^{\mathrm{i} \alpha-K z} \neq 0$ in $\overline{I I}$ which is fulfilled in case $\alpha=K \delta+\pi$ only if additionally $0<K \delta<\pi$. That is, the only solutions of (1) with the supposed behaviour at infinity are

$$
\therefore(x)=\frac{2 d K \varrho \sin K \delta \mathrm{e}^{-K x}}{1+\varrho^{2} \mathrm{e}^{-2 K x}+2 \varrho \mathrm{e}^{-K x} \cos K \delta}
$$

or putting $\varrho=\mathrm{e}^{K \beta}, \beta$ arbitrary real;

$$
u(x)=d K \frac{\sin K \delta}{\cos K \delta+\cosh K(x-\beta)}, \quad 0<K<\pi / \delta .
$$

These are (with $\beta=0$ ) the solutions of J. Satsuma and M: Mrmura [5] (see also [3, 4]).

2. Cauchy kernel. In the limiting case $\delta \rightarrow+\infty$ of (1) we have the equation

$$
d u_{x x}-(S u \cdot u)_{x}=0, \quad d>0
$$


where

$$
(S u)(x)=\frac{1}{\pi} \int_{-\infty}^{\infty} \frac{u(\xi)}{\xi-x} d \xi
$$

Again we are looking for sufficiently smooth solutions $u$ of (7) having vanishing limits $u( \pm \infty)=0$ and $u_{x}( \pm \infty) \equiv 0$ as $x \rightarrow \pm \infty$. The corresponding holomorphic function $w(z)=u+\mathrm{i} v$ in the upper half-plane $\Pi:-\infty<x<\infty, y>0$ with the boundary values $u(x, 0)=u(x)$ should be bounded at infinity. such that $v=0$ at infinity. Then

$$
w(z)=\frac{1}{\pi \mathrm{i}} \int_{-\infty}^{\infty} \frac{u(\xi)}{\xi-z} d \xi \quad \text { in } \Pi
$$

with the boundary values $v(x, 0)=-(S u)(x)$. Therefore integrating equation (7), we obtain the boundary condition $\operatorname{Re}\left[d w^{\prime}-(\mathrm{i} / 2) w^{2}\right]=0$ on $y=0$ for the function (8). Hence this function again obeys the differential equation (4).

As can be seen from above now for $K_{0} \neq 0$ no solutions to (4) of the required form exist. Therefore putting $K_{0}=0$ we get the differential equation

$$
\frac{w^{\prime}}{w^{2}}=\frac{\mathrm{i}}{2 d}
$$

with the general solution

$$
w(z)=\frac{2 d \mathrm{i}}{z-C}, \quad C \text { a complex number. }
$$

Putting $C=\alpha-\mathrm{i} \beta$ with $\beta>0$ and $\alpha$ arbitrary real, the function (9) is holomorphic in $\Pi$ and (Hölder) continuous in $\overline{I I}$ with $w(\infty)=0$. That is, we have the solutions

$$
u(x)=\frac{2 d \beta}{(x-\alpha)^{2}+\beta^{2}}, \quad \beta>0 \text {. }
$$

These are (with $\alpha=0$ ) again the solùtions of J. Sarsuma and M. Mrmura [5] (and $[3,4])$.

3. Hilbert kernel. In the case of periodic Solutions of (1) (with period $2 \pi$ ) the equation

$$
d u_{88}-(H u \cdot u)_{s}=0, \quad d>0,
$$

for $u=u(s)$ occurs, where

$$
(H u)(s)=\frac{1}{2 \pi} \int_{-\pi}^{\pi} u(\sigma) \cot \frac{\sigma-s}{2} d \sigma
$$

For sufficiently smooth solutions $u$ of (11) we introduce the Schwarz integral $W$, of $u$ (cf. [1: Chap. I, $\S 6]$ ). $W(z)=U+\mathrm{i} V$ is a holomorphic function in the unit disk $G:|z|<1$ with the boundary values $U\left(\mathrm{e}^{1 s}\right)=u(s)$ of $U$ and $V\left(\mathrm{e}^{1 s}\right)=-(H u)(s)$ of $V$ on the circumference $\Gamma: z=\mathrm{e}^{i s}$, where the conjugate harmonic function $V$ of $U$ satisfies the condition $V(0)=0$. Integrating equation (11); we obtain the boundary condition $\operatorname{Re}\left[\mathrm{i} d z W^{\prime}-(\mathrm{i} / 2) W^{2}\right]=0$ on $\Gamma$ for $W$. Therefore $W$ satisfies the differential equation

$$
d z W^{\prime}=\frac{1}{2} W^{2}+K_{0} \quad \text { in } G
$$

with a non-positive real constant $K_{0}\left(\doteq-(1 / 2) u^{2}(0)\right)$. 
As can be easily seen for $K_{0}=0$ no non-trivial regular solutions to (12) in $G$ exist: Therefore putting $K_{0}=.-(1 / 2) K^{2}, K>0$, we get the differential equation

$$
\frac{W^{\prime}}{W^{2}-K^{2}}=\frac{1}{2 d z}
$$

with the general solution

$$
W(z)=K \frac{1+C z^{K / d}}{1-C z^{K / d}}, \quad C \text { a complex number }
$$

This function represents a holomorphic function in $G$ with (Hölder) continuous boundary values on $\Gamma$ if $K=n d, n \in \mathrm{N}$, i.e.

$$
W(z)=n d \frac{1+. C z^{n}}{1-C z^{n}}
$$

with $|C|<1$ (cp. [2]). Putting $C=-\varrho \mathrm{e}^{-i n x}$ with $0<\varrho<1, \alpha$ arbitrary real, from (13) we obtain

or

$$
u(s)=\operatorname{Re} W\left(\mathrm{e}^{i \varepsilon}\right)=n d \frac{1-\varrho^{2}}{1+\varrho^{2}+2 \varrho \cos n(s-\alpha)}
$$

$$
u(s)=n d \frac{\sinh \varphi}{\cos n(s-\alpha)+\cosh \varphi}, \quad \varphi>0,
$$

where the parameter $\varphi$ is introduced by

$$
\cosh \varphi=\frac{1+\varrho^{2}}{2 \varrho} \quad \text { or } \quad \sinh \varphi=\frac{1-\varrho^{2}}{2 \underline{\theta}}
$$

These are (with $\alpha=0$ ) again the solutions of J. Satsuma and M. Memora [5] for the period $2 L=2 \pi$.

4. Variable diffusion coefficient. Finally, we consider the equation

$$
\left(d(s) u_{s}\right)_{s}-(H u \cdot u)_{s}=0
$$

for some special (positive) $2 \pi$-periodic functions $d$. If the eigenvalue problem

$$
u_{s}=\lambda(s) H u
$$

for a positive sufficiently smooth function $\lambda$ has a $2 \pi$-periodic (positive) solution $u_{0}$, then obviously $u_{0}$ is a steady state solution of the equation (15) with the diffusiou coefficient $d=u_{0} / \lambda$, The problem (16) is equivalent to the Steklov problem

$$
\partial \underline{V} / \partial r-\lambda(s) \underline{V}=0_{-} \quad \text { on } \Gamma_{-}, \ldots, V(0)=0,
$$

for the harmonic function $V$ in the unit disk $G: r<1$, which is the conjugate function to the Poisson integral $U$ of $u$. For instance, for $\lambda=k \in \mathbf{N}$ we have the eigensolutions $r^{k} \cos k s, r^{k} \sin k s$ of (17) and therefore the solution $u_{0}=a_{0}+a_{1} \cos k s$ $+a_{2}$ sin $k s$ with arbitrary constants $a_{0}, a_{1}, a_{2}$ of equation (15) for $d=d_{0}+d_{1} \cos k s$ $+d_{2} \sin k s$, where $a_{j}=k d_{j}, j=0,1,2$. In the particular case $d=d_{0}+d_{1} \cos s$ $+d_{2} \sin s$ with

$$
d_{0}>\sqrt{d_{1}^{2}+d_{2}^{2}}>0
$$


(insuring $d>0$ ) equation (15) as above leads to the complex differential equation

$$
\dot{A(z)} W^{\prime}=W^{2}-K^{2}
$$

for the holomorphic function $W$ in $G$, where $K$ is a complex constant and

$$
A(z)=2 z .\left[d_{0}+\frac{d_{1}}{2}\left(z+\frac{1}{z}\right)+\frac{d_{2}}{2 \mathrm{i}}\left(z-\frac{1}{z}\right)\right]
$$

is a holomorphic function in $G$. We write

$$
A(z)=\bar{D} z^{2}+2 d_{0} z+D=\bar{D}\left(z-z_{1}\right)\left(z-z_{2}\right)
$$

with $D=d_{1}+\mathrm{i} d_{2} \cdot$ and

$$
\dot{z_{1}}=\frac{1}{\bar{D}}\left[-d_{0}+\Delta\right] \in G, \quad z_{2}=\frac{1}{\bar{D}}\left[-d_{0}-4\right] \oplus \bar{G},
$$

where $\Delta^{\prime}=\sqrt{d_{0}^{2}-|D|^{2}}>0$ by (18). That is, $W$ satisfies the differential equation

$$
\frac{W^{\prime}}{W^{2}-K^{2}}=\frac{1}{\bar{D}\left(z-z_{1}\right)\left(z-z_{2}\right)} \quad \text { in } G \text {. }
$$

As can be easily seen for $K=0$ no non-trivial regular solutions to (19) in $G$ exist. For $K \neq 0$ we have the general solution

$$
W(z)=K \frac{1+C \zeta^{K / \Delta}}{1-C \zeta^{K / \Delta}}, \quad \zeta=\frac{z-z_{1}}{z-z_{2}}, C \text { a complex number }
$$

This function represents a holomorphic function in $G$ with (Hölder) continuous boundary values on $\Gamma$ if $K=\dot{n} \Delta, \dot{n} \in \dot{\mathbf{N}}$, i.e. putting $C=C_{0}{ }^{n}$,

$$
W(z)=n \Delta \frac{\left(z-z_{2}\right)^{n}+C_{0}^{n}\left(z-z_{1}\right)^{n}}{\left(z-z_{2}\right)^{n}-C_{0}^{n}\left(z-z_{1}\right)^{n}}
$$

with the restriction on $C_{0}$ that $\left(z-z_{2}\right)^{n} \neq C_{0}^{n}\left(z-z_{1}\right)^{n}$ for $|z| \leqq 1$, i.e.

$$
\cdot\left|z_{2}-C_{0} \mathrm{e}^{\mathrm{i} \frac{2 k \pi}{n}}\right|>\left|1-C_{0} \mathrm{e}^{\mathrm{i} \frac{2 k \pi}{n}} z_{1}\right|, \quad 0 \leqq k \leqq n-1 \text {. }
$$

Since $\left|z_{2}\right|>1$, the condition (21) is fulfilled for sufficiently small $\left|C_{0}\right|$. From (20) the steady state solutions $u$ of (15) are given by

$$
u(s)=n \Delta \frac{\left|\mathrm{e}^{1 s}-z_{2}\right|^{2 n}-\left|C_{0}\right|^{2 n}\left|\mathrm{e}^{i s}-z_{1}\right|^{2 n}}{\left|\left(\mathrm{e}^{\mathrm{i} s}-z_{2}\right)^{n}-C_{0}{ }^{n}\left(\mathrm{e}^{1 s}-z_{1}\right)^{n}\right|^{2}}, \quad n \in \mathbf{N}
$$

Especially for $n=1$ with $C_{0}=1$ we retrieve the above solution $u_{0}=d$.

\section{REFERENCES}

[1] ГАхов, Ф. Д.: Краевьіе задачи (2-ое изд.). Москва: Физматгиз 1963. Engl. transl.: Gaknov, F. D.: Boundary Value Problems. Oxford: Pérgamon Press 1966.

[2] Gerlach, W., and L. v. Wolfersdohf: On a nonlinear nonlocal diffusion equation of Satsima and Mimura. Complex Variables 9 (1987), 81-89. 
[3]. Mrmura, M.: Some convection-diffusion equations arising in population dynamics. Contemp. Math. 17 (1983), 343-351.

[4] Satsoma, J.: Exact solutions of a nonlinear diffusion equation. J. Phys. Soc. Japan (1981), 1423-1424.

[5] Satsuma, J:, and M. Mmora: Exact treatment of nonlinear diffusion equations with. singular integral terms. J. Phys. Soc. Japan 54.(1985), 894-900.

[6] Woons, L. C.: The Theory of Subsonic. Plane Flow. Cambridge: Univ. Press 1961.

Manuskripteingang: 13.02.1987

\section{VERFASSER :}

Prof. Dr.-Lothar v. Wolfersdorf

Sektion Mathematik der Bergakademie Freiberg Bernhard-v.-Cotta-Str. 2

DDR - 9200 Freiberg . 